\title{
Comparison of the safety of drug delivery via HFA- and CFC- metered dose inhalers in CAO
}

\author{
G. Huchon*, P. Hofbauer**, G. Cannizzaro***, P. lacono ${ }^{+}$, F. Wald ${ }^{++}$
}

Comparison of the safety of a fenoterol/ipratropium bromide combination delivered by HFA-MDI or CFC-MDI in chronic airways obstruction. G. Huchon, P. Hofbauer, G. Cannizzaro, P. Iacono, F. Wald. (C)ERS Journals Ltd 2000.

ABSTRACT: The objective of this study was to compare the long-term safety of a fixed combination of fenoterol hydrobromide $(50 \mu \mathrm{g})$ and ipratropium bromide $(20$ $\mu \mathrm{g})$ delivered using a metered dose inhaler (MDI) formulated with a non-chlorinated propellant, hydrofluoroalkane134a (HFA-MDI), with delivery using the conventional chlorofluorocarbon propellant (CFC-MDI, Berodual $\mathbb{R} /$ Bronchodual $\mathbb{R}$ ).

The study was designed according to Safety Assessment of Marketed Medicines (SAMM) guidelines, to reflect as far as possible the use of MDIs under normal prescribing conditions. Two thousand and twenty-seven patients with chronic airways obstruction (CAO) were enrolled from 99 centres in France, 95 centres in Germany and 24 centres in Italy. Following a 2 -week run-in period, patients were randomized on a 2:1 basis (1,348 patients to HFA-MDI, 679 patients to CFC-MDI) to receive a flexible dose regimen of the combination ( 2 puffs, $2-4$ times a day, as prescribed by the investigator) during a 12-week open label phase.

The overall incidence of adverse events was comparable between both groups. In addition, the incidence of respiratory side effects was also similar, with CAO exacerbations or bronchitis the most frequently recorded events.

The safety profile of the HFA formulation was comparable to those of the marketed CFC-MDIs used in Germany and France/ltaly.

No clinically significant differences were detected between HFA134a or CFC driven inhalers on the switch from CFC- to HFA-MDI (2 weeks before randomisation versus 2 weeks after randomization).

There was a trend for taste complaints to be reported more frequently by patients in the HFA-MDI group ( $0.7 \%$ before randomization versus $3.4 \%$ after randomization). This, however, was an expected finding as the HFA134a formulation does have a different taste to the CFC formulation.

No difference between formulations was observed in the incidences of coughing or paradoxical bronchospasm. The incidence of falls in FEV1 $>15 \%$ within 15 min following inhalation at each of the clinic visits was $1.2 \%$ for both CFC- and HFAMDIs.

In conclusion, administration of a fenoterol/ipratropium bromide combination via hydrofluoroalkane-metered dose inhaler is as safe as delivery by the currently available chlorofluorocarbon-metered dose inhaler, in an extended population of patients with CAO under normal prescribing conditions.

Eur Respir J 2000; 15: 663-669.

\begin{abstract}
*Service de Pneumologie, Hôpital de l'Hotel-Dieu, Paris, France. **Arzt für Innere Medizin, Dürreplatz 4, 69469 Weinheim, Germany. ***Divisione di Pneumologia, Ospedale di Cittadella, Via Riva Ospedale, 35013-Cittadella (PD), Italy. ${ }^{+}$Boehringher Ingelheim, Reims, France ${ }^{++}$Boehringer Ingelheim KG, Ingelheim, Germany.
\end{abstract}

Correspondence: G. Huchon, Service de Pneumologie et de Réanimation, Hôpital de l'Hôtel Dieu, 1 Place du Parvis de Notre-Dame, F-75004 Paris, France. Fax: 33142348448

\section{Keywords: Asthma}

chlorofluorocarbon

chronic obstructive pulmonary disease hydrofluoroalkane

ipratropium

metered dose inhaler

Received: December 191999

Accepted after revision December 311999
Metered dose inhalers (MDIs) have been used in the treatment of obstructive airway diseases, including asthma and chronic obstructive pulmonary disease (COPD), for several decades. However, these devices use chlorofluorocarbon (CFC) propellants which are being withdrawn in response to environmental concerns [1]. Therefore, alternative propellants or delivery devices are currently in clinical development, such as non-CFC MDIs, dry powder inhalers and a propellant-free soft mist inhaler [2-4].

The hydrofluoroalkane (HFA), HFA134a $(1,1,1,2-$ tetraflouroethane) is a possible replacement for CFC propellants in MDIs. Toxicity trials in animals have shown that HFA134a is an inert and nontoxic substance. Studies in healthy volunteers have demonstrated that inhalers formu- lated with HFA134a are as safe and well tolerated as CFC driven MDIs [5-7].

The objective of the present study was to compare the long-term safety of fenoterol hydrobromide and ipratropium bromide (at a fixed dose combination of 50 and 20 $\mu \mathrm{g}$ per puff, respectively) delivered by a reformulated MDI containing the HFA134a propellant (HFA-MDI), with delivery by the standard CFC-driven MDI (CFC-MDI, Bero-

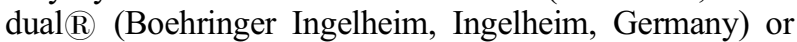
Bronchodual $\mathbb{R}$ (Boehringer Ingelheim, Reims, France), in patients with chronic airways obstruction (CAO). Therapeutic equivalence of the two formulations has been established previously [8-10]. However, this trial was designed primarily to assess safety according to Safety Assessment 
of Marketed Medicines (SAMM) guidelines [11], to reflect the use of HFA-MDIs under normal prescribing conditions.

\section{Materials and methods}

\section{Patients}

Patients eligible for the study were outpatients aged 1880 yrs with CAO including asthma, COPD or mixed conditions as partly defined by the American Thoracic Society [12]. For the purposes of this trial, asthmatics were defined a posteriori as patients aged $<45 \mathrm{yrs}$, with a smoking history of $<10$ pack-yrs and a history of bronchial reactivity to allergens or dust. COPD patients were defined a posteriori as $>45 \mathrm{yrs}$, with a smoking history of $>20$ pack-yrs, and no history of bronchial reactivity to allergens nor dust and no history of allergy. Other patients were categorized as having mixed conditions. At the initial screening visit, each patient provided written informed consent and underwent a complete medical examination to fulfil inclusion and exclusion criteria.

To be included in the study, patients had to have stable CAO with no hospital admission for an exacerbation and no major change in medication for at least 4 weeks prior to the screening visit. Additionally, all patients were required to have an initial forced expiratory volume in one second (FEV1) of $\geq 40 \%$ of the predicted value when not receiving bronchodilator medication, according to the tables for standardized lung function tests of the European Community for Coal and Steel [13]. In an attempt to study patients under as normal conditions as possible, exclusion criteria were limited to the contraindications listed for the marketed products in the data sheet or summary of product characteristics. Consequently, subjects were excluded if they had a respiratory tract infection, tuberculosis, cancer or were intolerant to the study drugs or excipients. Oral steroids, inhaled steroids, theophyllines, cromoglycate, nedocromil, long-acting oral and long-acting inhaled $\beta_{2^{-}}$ agonists were allowed throughout the study, provided that the dosage remained stable. Patients were instructed to take this medication at the same time each day. In addition, if a spacer device was used before the trial, it also had to be used during the trial. It was possible that patients were using Berodual $\mathbb{R}$ (Boehringer Ingelheim)/ Bronchodual@ (Boehringer Ingelheim) before the trial. The following drugs were not allowed during the study and had to be withheld before the start of the screening visit: all other investigational drugs, other inhaled shortacting $\beta_{2}$-agonists, oral short-acting $\beta_{2}$-agonists, and other inhaled anticholinergics. Patients were also not eligible if they used $>12$ puffs $\cdot$ day $^{-1}$ of Berodual $\mathbb{R}$ (Boehringer Ingelheim)/Bronchodual (B) (Boehringer Ingelheim) during the run-in phase of the study. If symptoms were not relieved with the dosage regimen prescribed by the investigator, use of additional puffs of the study drug was allowed as rescue medication. During the study, temporary increases or addition of bronchodilators, steroids, antibiotics or theophylline were allowed in the case of an exacerbation of CAO, which had to be treated normally.
If the exacerbation lasted $>14$ days, the patient had to be withdrawn from the study.

A total of 2,211 eligible patients were screened and participated in the run-in period of the trial and 2,027 were randomized and included in the 12 week study phase. The study was designed so that the proportion of patients receiving each of the treatments during the study phase was unbalanced: $1,348(66.5 \%)$ were randomized to HFA-MDI and $679(33.5 \%)$ to CFC-MDI. Patient characteristics, medical history and baseline features were similar between the two treatment groups (table 1). The overall trial population was composed of 1,162 males and 865 females of mean \pm SD age $54 \pm 15$ yrs with a median duration of CAO of 8 yrs. In terms of pulmonary function, the mean FEV1 of the overall population was $2.05 \pm 1.75 \mathrm{~L}(69.3 \pm 19.8 \%$ pred). A total of 1,265 patients were randomized and entered into the trial from Germany, and 466 and 296 patients were included in the 12 week trial phase from France and Italy, respectively. During the study phase the drop-out rate was lower in France and Germany (6.2 and $7.0 \%)$ than that in Italy $(13.5 \%)$. Between the HFA and CFC formulations there was a similar drop-out rate due to adverse events $(4.1 \%$ in the HFA group versus $3.7 \%$ in the CFC group) and due to lack of efficacy (0.4\% HFA-MDI versus $0.3 \%$ CFC-MDI). The overall drop-out rate was $7.8 \%$. As a result of drop-outs, a total of 1,176 patients from Germany, 437 patients from France and 256 patients from Italy completed the 12 week trial phase.

The study was carried out in accordance with the principles of the declaration of Helsinki and approved by the local Ethics Committees.

Table 1. - Patient demographics and baseline characteristics $(n=2,027)$

\begin{tabular}{lcc}
\hline & HFA-MDI & CFC-MDI \\
\hline $\mathrm{n}$ & 1348 & 679 \\
Age yrs & $54 \pm 15$ & $55 \pm 16$ \\
$\begin{array}{l}\text { Male/female } \\
\text { Median duration of disease yrs }\end{array}$ & $795 / 553$ & $367 / 312$ \\
$\begin{array}{l}\text { Smoking history \% } \\
\text { Smokers }\end{array}$ & $19-66)$ & $9(0-57)$ \\
Exsmokers & 33.2 & 18.3 \\
Never smokers & 47.4 & 32.8 \\
CAO* & & 48.9 \\
Asthma \% & 19.7 & 19.4 \\
COPD \% & 10.9 & 9.9 \\
Mixed conditions \% & 69.4 & 70.7 \\
Baseline FEV1 L & $2.06 \pm 0.79$ & $2.03 \pm 0.79$ \\
Baseline FVC L & $2.93 \pm 0.98$ & $2.90 \pm 1.01$ \\
Baseline FEV1 \% pred & $69.2 \pm 20.04$ & $69.7 \pm 18.7$ \\
$\begin{array}{l}\text { FEV1 reversibility at 5 min } \\
\text { post-dose \% }\end{array}$ & $12.6 \pm 21.9$ & $12.4 \pm 15.4$ \\
& & \\
\hline
\end{tabular}

Data are presented as mean $\pm \mathrm{SD}$, or median with range in parentheses, or as absolute numbers. *: Chronic airways obstruction (CAO) profile of the study population defined as: 1) asthma age $<45$ yrs, smoking history $<10$ pack yrs, history of bronchial reactivity to allergens or dust; 2) chronic obstructive pulmonary disease (COPD) age $>45$ yrs, smoking history $>20$ pack-yrs, no history of bronchial reactivity to allergens or dust and no history of allergy; and 3) other patients categorized as mixed conditions. HFA: hydrofluoroalkane; MDI: metered dose inhaler; CFC: chlorofluorocarbon; FEV1: forced expiratory volume in one second; FVC: forced vital capacity. 


\section{Study design}

This study was an open-labelled, randomized trial performed in 218 centres in France, Germany and Italy using an unbalanced parallel group design. After recruitment, patients entered a 2 -week run-in period where they received two puffs of fenoterol hydrobromide $(50 \mu \mathrm{g})$ and ipratropium bromide $(20 \mu \mathrm{g})$ via CFC-MDI 2-4 times daily. On completion of the run-in period, eligible patients were randomly assigned to continue with the combination delivered via CFC-MDI or switched to delivery by HFA-MDI at two puffs 2-4 times daily for 12 weeks, as prescribed by the investigator. Each patient was instructed to inhale the study drug at the same time throughout the trial. Four clinic visits were made during the trial: screening, inclusion and at weeks 6 and 12 of the 12-week study phase. At each visit, patient's pulmonary function at pre- and 5-min postdose (to detect paradoxical falls in FEV1), in addition to vital signs and adverse events, were evaluated.

As the excipients in the marketed Berodual $\AA$ (Boehringer Ingelheim)/Bronchodual $\mathbb{R}$ ) (Boehringer Ingelheim) CFC-MDIs differ in Europe, the CFC formulations used during the trial were identical to those available in the participating countries. Consequently, a CFC-MDI containing a sorbitan trioleate (SPAN) tensioactive agent was used in Germany and a CFC-MDI containing soya-lecithin was used in France and Italy.

\section{Methods}

The safety profile of both formulations was assessed from diaries and patient self-reporting, which were recorded by the investigator at each clinic visit during the 12 week study phase. The primary end-point was the incidence of adverse events with particular attention given to the frequency of coughing, wheezing, and bronchospasm following inhalation of study medication. Bronchospasm was defined as an adverse event only if it was reported by the patient, there was evidence of related clinical abnormalities, a therapeutic action was needed, or there was a $>15 \%$ fall in FEV1 below baseline within 15 min following inhalation of the test drug. Secondary endpoints included the use of additional puffs of study drug as rescue medication and the number of CAO exacerbations.

Pulse rate and blood pressure were measured before lung function testing on all four visits. Clinical examinations were carried out upon admission and completion of the study, with any abnormal clinical findings recorded by the investigator.

Pulmonary function (FEV1) and forced vital capacity (FVC) were measured by spirometry at baseline and $5 \mathrm{~min}$ following administration of the treatment on each test day (visits 2, 3 and 4) according to American Thoracic Society snowbird standards [14]. Patients were eligible if FEV1 was $\geq 40 \%$ pred. Three FEV1 and FVC measurements were taken and the highest value recorded in each case. The aim of these measurements was to detect paradoxical falls in FEV1 rather than to determine the efficacy of both formulations. Lung function tests were performed at the same time of day at each visit to allow for diurnal variation and patients were asked to avoid strenuous exercise for $12 \mathrm{~h}$ before testing.
Analysis

The null hypotheses assumed that the incidence of adverse events before and after randomization would not differ, and that the outcome of the randomized trial phase was equal in the two treatment groups. The experimental treatments were allocated in an unbalanced fashion: two thirds of all patients were included in the HFA-MDI group versus one third of patients in the CFC-MDI group. This randomization exposed a large number of patients to the HFA-MDI formulation to replicate conditions in clinical practice and to increase the power of the study so that even adverse events occurring with low incidence rates could be detected. A sample size of 1,000 HFA-MDI patients was required to detect a $0.3 \%$ incidence rate of adverse events at a two-sided level of significance with a $95 \%$ power. Since 1,348 patients were eventually included in the HFAMDI group a power of $98 \%$ was reached to detect incidence rates of $0.3 \%$. All safety analyses were performed with the intent-to-treat dataset using data available from patients on a given test day. Safety data were analysed using analysis of covariance (ANCOVA). The incidence of adverse events between treatment groups was compared using either McNemar tests for ordinal categorical variables or Fisher's exact test for other categorical variables. Due to the large number of centres, the statistical tests were not adjusted for centres and in the linear model "centre" was considered to be a random factor.

FEV1 and FVC original measurements and the differences (post-dose minus pre-dose) were described by summary statistics (per treatment group, visit and time) and displayed graphically by mean time response curves using the least square mean \pm SEM (obtained from the ANCOVA described below).

The pre-dose values of weeks 6 and 12 were analysed using an ANCOVA with "day 0 pre-dose value" as covariate, fixed factor "treatment" and random factors "centre" and "centre treatment" interaction. Estimates for treatment differences and the respective $95 \%$ confidence intervals were tabulated.

The post-dose values of day 0 , week 6 and week 12 were analysed using an ANCOVA with the respective "test day pre-dose value" as covariate, fixed factor "treatment" and random factors "centre" and "centre treatment" interaction. Estimates for treatment differences and the respective $95 \%$ confidence intervals were tabulated.

\section{Results}

\section{Overall safety analysis}

The incidence of adverse events in the 2,027 randomized patients was comparable between the two treatment groups with $36.4 \%$ (491 patients) in the HFA-MDI group and $37.1 \%$ (252 patients) in the CFC-MDI group reporting at least one adverse event during the randomized phase (table 2). In addition, the rates of potential systemic effects of the trial drug, based on the incidence of cardiovascular events, mouth dryness or tremor, were balanced across both formulations. The most frequently reported adverse events were respiratory disorders including asthma or COPD exacerbations, bronchitis, cough and dyspnoea (table 3 ). There were no statistically significant differences between formulations for each of the most 
Table 2. - Percentage of patients experiencing adverse events during the 12-week treatment period

\begin{tabular}{lrr}
\hline & HFA-MDI & CFC-MDI \\
\hline $\mathrm{n}$ & 1348 & 679 \\
All adverse events & $36.4(8.2)$ & $37.1(5.6)$ \\
General disorders (all) & $8.8(0.6)$ & $7.7(0.4)$ \\
Headaches & $1.9(0.2)$ & $1.6(0.3)$ \\
Cardiovascular & $1.2(0.2)$ & $0.9(0.3)$ \\
Heart rate and rhythm disorders & $0.6(0.2)$ & $1.0(0.6)$ \\
Nervous system (all) & $3.8(1.3)$ & $3.2(0.7)$ \\
Dizziness & $0.7(0.3)$ & $0.9(0.1)$ \\
Tremor & $0.4(0.4)$ & $0.4(0.1)$ \\
Gastrointestinal (all) & $5.2(1.0)$ & $6.3(0.7)$ \\
Dry mouth & $0.4(0.3)$ & $0.6(0.3)$ \\
Respiratory disorders (all)* & $21.7(4.4)$ & $21.2(2.4)$ \\
Taste complaints & $2.0(1.9)$ & $1.2(1.0)$ \\
\hline
\end{tabular}

HFA: hydrofluoroalkane; MDI: metered dose inhaler; CFC: chlorofluorocarbon. Data are presented as means with the percentage considered to be drug related in parentheses. *: includes all upper and lower respiratory tract adverse events.

clinically important respiratory adverse events; except for COPD exacerbation $(4.1 \%$ in the CFC-MDI group versus $2.4 \%$ in the HFA-MDI group, $\mathrm{p}=0.04$ ) and sputum increase $(1.3 \%$ CFC-MDI versus $0.4 \%$ HFA-MDI, $\mathrm{p}=0.02)$. The intensity and duration of the adverse events were similar in both treatment groups.

Paradoxical bronchospasm was experienced by just one patient during the run-in period of the trial. The rate of falls of $>15 \%$ in baseline FEV1 within 15 min following inhalations at clinic visits was $1.2 \%$ for both CFC- and HFA-MDIs.

Effect of different excipients in chlorofluorocarbon-metered dose inhalers

The incidence of adverse events between the HFA formulation and the two different CFC-MDI excipients used in Germany (SPAN) and France/ltaly (soya-lecithin) was comparable. The frequency of adverse events was similar for each country, except for respiratory disorders (31.6\% in France/ltaly versus $15 \%$ in Germany).

\section{Switch effect analysis}

The frequency of adverse events over the 2 week run-in period before inclusion (CFC-MDI) compared with the incidence of adverse events over the 2 weeks following inclusion (randomized to HFA- and CFC-MDIs) was similar between treatments (table 4). Incidences of asthma/ COPD exacerbations and bronchitis increased slightly after randomization in both treatment groups. In contrast, there was a trend for taste complaints to be reported more frequently when patients switched to HFA-MDI $(0.7 \%$ before randomization versus $3.4 \%$ after randomization). Although no statistical differences were detected between formulations, further analysis of the HFA-MDI group showed that taste complaints tended to be reported more often by female patients and asthmatics than by male patients or patients with COPD or mixed conditions.

Deaths, serious adverse events and adverse events leading to withdrawal

One patient died during the run-in period of the trial (lung cancer) and five patients died during the randomized phase: four $(0.3 \%)$ of the 1,348 patients in the HFA-MDI group (one patient from a heart attack, one patient from a myocardial infarction and two patients from cardiac infarctions) and one $(0.1 \%)$ of the 679 patients in the CFC-MDI group from a cerebral stroke.

There was no difference between the two treatment groups in the incidence of serious adverse events and adverse events leading to withdrawal. During the 12 week study period 36 patients $(2.7 \%)$ in the HFA-MDI group and $25(3.7 \%)$ patients in the CFC-MDI group reported serious adverse events. Only two serious adverse events were considered drug-related: episodes of tachyarrythmia and bronchoconstriction. Both occurred during the run-in period and the two patients were not randomized. Fiftyeight $(4.3 \%)$ of the 1,348 patients randomized to the HFA134a formulation were withdrawn from the study compared with $27(4.0 \%)$ of the 679 patients randomized to the CFC-MDI. None of the adverse events leading to patient withdrawal in both treatment groups were taste complaints.

\section{Other safety variables}

The use of rescue medication, defined as extra puffs of the study drug, was similar in each treatment group (fig. 1). For both study groups, the percentage of patients using rescue medication decreased after weeks 1 and 7 of the 12

Table 3. - Number of patients experiencing respiratory adverse events during the 12-week treatment period

\begin{tabular}{|c|c|c|c|c|c|c|}
\hline & \multicolumn{3}{|c|}{ HFA-MDI (n=1348) } & \multicolumn{3}{|c|}{ CFC-MDI (n=679) } \\
\hline & $\mathrm{n}$ & $\%$ & $\begin{array}{l}\% \text { considered to } \\
\text { be drug-related }\end{array}$ & $\mathrm{n}$ & $\%$ & $\begin{array}{l}\% \text { considered to } \\
\text { be drug-related }\end{array}$ \\
\hline $\mathrm{n}$ & 1348 & 679 & & 1348 & 679 & \\
\hline Asthma exacerbation & 48 & 3.6 & 0.1 & 27 & 4.0 & 0.1 \\
\hline COPD exacerbation* & 33 & 2.4 & 0.4 & 28 & 4.1 & 0.4 \\
\hline Bronchitis & 49 & 3.6 & 0.07 & 35 & 5.2 & 0.0 \\
\hline Coughing & 50 & 3.7 & 2.2 & 20 & 2.9 & 1.2 \\
\hline Increased sputum** & 5 & 0.4 & 0.0 & 9 & 1.3 & 0.0 \\
\hline Dyspnoea & 46 & 3.4 & 0.7 & 15 & 2.2 & 0.4 \\
\hline Bronchospasm & 8 & 0.6 & 0.0 & 2 & 0.3 & 0.0 \\
\hline
\end{tabular}

*: $\mathrm{p}=0.04 ; * *: \mathrm{p}=0.02$, Fisher's exact test. HFA: hydrofluoroalkane; MDI: metered dose inhaler; CFC: chlorofluorocarbon; COPD: chronic obstructive pulmonary disease. 
Table 4. - Percentage of patients with adverse events before and after inclusion

\begin{tabular}{|c|c|c|c|c|}
\hline & \multicolumn{2}{|c|}{ HFA-MDI } & \multicolumn{2}{|c|}{ CFC-MDI } \\
\hline & Before & After & Before & After \\
\hline $\mathrm{n}$ & 1348 & 679 & 1348 & 679 \\
\hline $\begin{array}{l}\text { Number of patients with } \\
\text { at least one adverse event }\end{array}$ & 14.8 & 13.8 & 14.4 & 12.7 \\
\hline $\begin{array}{l}\text { Number of patients with } \\
\text { at least one respiratory adverse event }\end{array}$ & 6.3 & 7.3 & 6.6 & 6.3 \\
\hline Asthma exacerbation* & 0.7 & 1.9 & 1.5 & 2.7 \\
\hline COPD exacerbation* & 1.0 & 1.6 & 0.3 & 1.5 \\
\hline Bronchitis* & 0.5 & 1.6 & 0.0 & 2.1 \\
\hline Coughing* & 2.7 & 3.9 & 3.2 & 3.2 \\
\hline Dyspnoea* & 3.1 & 1.2 & 5.0 & 1.2 \\
\hline Tremor* & 1.7 & 0.3 & 1.8 & 0.3 \\
\hline Dry mouth* & 1.8 & 0.5 & 1.8 & 0.6 \\
\hline Taste complaints* & 0.7 & 3.4 & 1.2 & 1.2 \\
\hline
\end{tabular}

HFA: hydrofluoroalkane; MDI: metered dose inhaler; CFC: chlorofluorocarbon; COPD: chronic obstructive pulmonary disease. *: percentages correspond to the ratio of number of adverse events to the number of patients in the group with one patient possibly having reported more than one adverse event.

week study phase. There were no clinically significant changes in blood pressure or pulse rate following inhalation of fenoterol hydrobromide and ipratropium bromide from either inhaler type. Comparisons between physical examinations carried out at the screening visit and after completion of the study showed no differences.

\section{Efficacy}

Analysis of FEV1 and FVC showed that a fixed combination dose of fenoterol/ipratropium bromide delivered via HFA-MDI produced a comparable efficacy profile to delivery by CFC-MDI. Pre-dose FEV1 remained constant during the 12-week treatment period for both HFA and CFC patients. The reversibility of FEV1 at $5 \mathrm{~min}$ following treatment with the HFA formulation was similar to that observed with the CFC formulation, confirming the comparable bronchodilator effect produced from both types of inhaler (fig. 2). This was also supported by $5 \mathrm{~min}$ post-dose reversibility tests using analyses of the FVC.

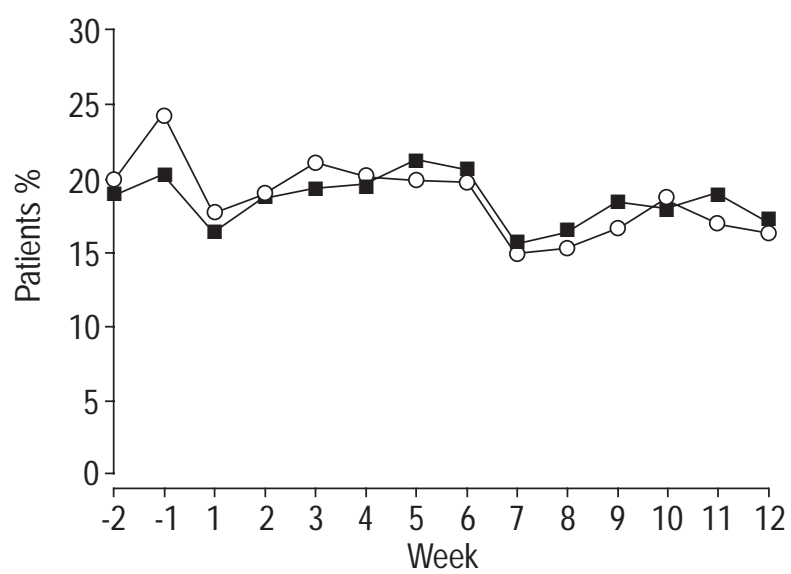

Fig. 1. - Percentage of patients using rescue medication throughout the trial $(n=2,027)$. $\square$ : hydrofluoroalkane-metered dose inhaler; $\bigcirc$ : chlorofluorocarbon metered dose inhaler.

\section{Discussion}

Due to the harmful effects of CFCs on the environment, alternatives to freon-driven MDIs are required for inhalation therapy. However, the use of CFC-containing MDIs is now well established in patients with respiratory disease and there may be concerns about switching to new inhalation devices. Therefore, it is important that patients and physicians are assured that alternatives, such as HFA134a, are at least equivalent to CFC-MDIs in terms of efficacy and safety.

In contrast to the normal trial design employed for a respiratory drug or device, this study was designed to replicate normal prescribing conditions, in an attempt to closely reflect what will happen when HFA-MDIs are introduced to the market. This included the recruitment of a large extended population of patients with CAO to be as representative as possible of the general population of users. In addition, the CFC-MDIs used in the trial were identical to those used in the participating country.

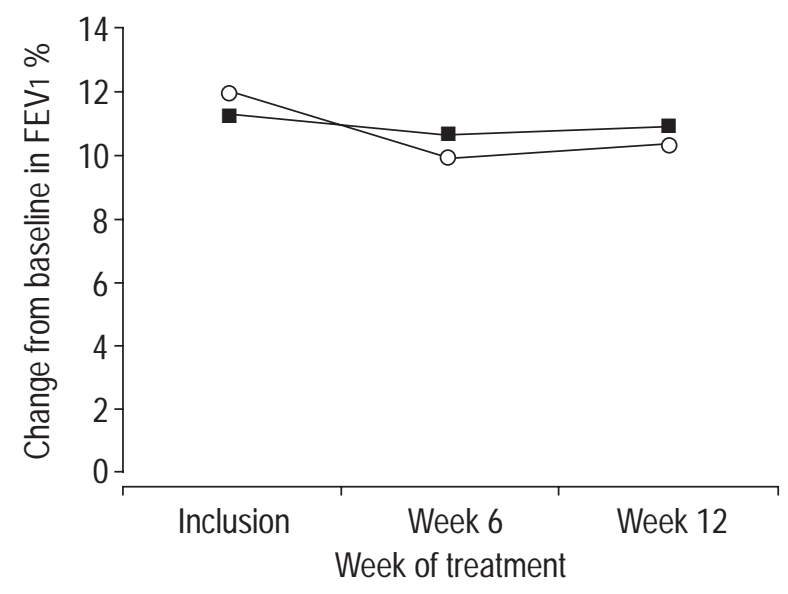

Fig. 2. - Mean forced expiratory volume in one second (FEV1) reversibility from pre-dose $5 \mathrm{~min}$ after inhalation of fenoterol hydrobromide and ipratropium bromide by hydrofluoroalkane (HFA)- or chlorofluorocarbon (CFC)-metered dose inhaler (MDI) $(n=1,659)$. HFA-MDI; O: CFC-MDI. 
Specifically, a SPAN CFC-MDI was used in Germany and a soya-lecithin CFC-MDI was used in France/Italy. The results demonstrate that administration of a combined dose of fenoterol hydrobromide and ipratropium bromide (50 and $20 \mu \mathrm{g}$ per puff, respectively) via HFA-MDI is as safe as delivery by CFC-MDI in patients with CAO.

The overall incidence of adverse events was similar between formulations. Respiratory disorders including events such as asthma and dyspnoea were largely comparable between both groups of patients. However, there were higher incidences of COPD exacerbations and sputum increases reported by patients in the CFC group. The reasons for the higher frequencies are unclear and are probably chance findings. The urge to cough during the study was similar in both populations. None of the randomized patients reported episodes of wheezing or paradoxical bronchospasm, and the number of patients showing a fall in baseline FEV1 was comparable between treatments.

Analysis of the incidence rate of adverse events between HFA-MDIs and the two different CFC excipients used in Germany and France/ltaly showed that the HFA formulation was comparable to both CFC-MDIs. Although the incidence rate of adverse events was similar in Germany and France/ltaly for both formulations, respiratory disorders were reported more frequently in France/ltaly $(31.7 \%)$ than in Germany $(15.3 \%)$. This finding may be explained by the inclusion of fewer COPD patients and a lower frequency of heavy smokers from Germany. Another possible explanation is that different types of centres were used in the participating countries. In Germany, many investigators were general practitioners while in France/Italy, they were exclusively chest physicians either in private hospitals or public hospitals. This may have resulted in contrasts in the reporting of respiratory adverse events between investigators.

There were no differences between formulations on the switch from CFC- to HFA-MDI. There was a trend for taste complaints to be reported more frequently by patients in the HFA-MDI study group $(0.7 \%$ versus $3.4 \%)$. Furthermore, this taste difference tended to be reported more often by the asthmatic patients than patients with COPD or mixed conditions, perhaps reflecting the increased sensitivity of asthmatics to medication changes. Female patients also tended to report taste differences with the new formulation more frequently than male patients. It is important to note, however, that previous studies have shown that taste complaints associated with the HFA134a formulation should not affect general acceptance of this new type of inhaler.

The use of rescue medication throughout the trial was comparable between both groups of patients. Interestingly, the proportion of patients using rescue medication decreased after clinic visits in weeks 1 and 7 of the study phase. This probably reflects improvements in the dosing regimen prescribed by the investigators and patient experience with the study treatments. In terms of vital signs and physical examination, the study indicated that there is no clinically relevant difference in tolerability between MDIs formulated with HFA134a or CFC propellants.

Pulmonary function measurements were performed as 5 min post-dose reversibility tests at each visit throughout the 12-week treatment period, to detect paradoxical falls in FEV1. The early bronchodilator effect of fenoterol hydrobromide and ipratropium bromide administered via HFA- or CFC-MDIs was comparable and stable throughout the study in patients with CAO.

These results are in agreement with those previously reported for the reformulated fenoterol/ipratropium bromide inhaler. In a dose response study, MAESEN et al. [8] compared the bronchodilator effect from 0-360 min following delivery of the two inhaler formulations in 52 patients with asthma. Both formulations were therapeutically equivalent at the standard dose of $100 \mu \mathrm{g}$ fenoterol/ $40 \mu \mathrm{g}$ ipratropium bromide in improving peak FEV1 and average FEV1. In addition, the time to onset and duration of efficacy were also comparable between the marketed product and the HFA-MDI. The long-term safety and efficacy of the novel HFA inhaler was assessed further in two 12-week studies. In one of the studies, reported by Aubier et al. [9], the safety profiles of both formulations, delivering a $100 / 40 \mu \mathrm{g}$ fenoterol/ipratropium bromide dose four times daily, was compared in 250 adult patients with either asthma or COPD. The second study, reported by REFABERT et al. [10], evaluated the safety of a three times daily dosing regimen in 169 asthmatic children (mean age 10.3 yrs). Both studies showed that delivery by HFA-MDI produced a comparable bronchodilator effect to the marketed MDI. In the study using adult patients, equivalent efficacy, with no clinically significant differences, was observed for both formulations. Similarly, in the trial with asthmatic children, the efficacy profile of the two inhaler devices was comparable in terms of the reversibility of FEV1 and the morning and evening peak expiratory flow measurements. Analysis of the safety end-points showed that the HFA- and CFC-MDIs had similar safety profiles.

In conclusion, the results of this study show that administration of a fenoterol/ipratropium bromide combination by a metered dose inhaler formulated with hydrofluoroalkane $134 \mathrm{a}$ is as safe as administration by a chlorofluorocarbon-containing metered dose inhaler. The use of hydrofluoroalkane $134 \mathrm{a}$ as a propellant for Berodual $\mathbb{R}$ (Boehringer Ingelheim)/Bronchodual@ (Boehringer Ingelheim) is, therefore, a suitable alternative to the currently available chlorofluorocarbon-driven inhaler.

\section{References}

1. Pavia D, McLeod L. The environmental impact of inhaled aerosols. Eur Respir Rev 1994; 4: 75-77.

2. Partridge M, Woodcock A. Metered dose inhalers free of chlorofluorocarbons. BMJ 1995; 310: 684-685.

3. Pavia D. Efficacy and safety of inhalation therapy in chronic obstructive pulmonary disease and asthma. Respirology 1997; 2 (Suppl. 1): S5-S10.

4. Wetterlin K. Turbuhaler: a new powder inhaler for administration of drugs to the airways. Pharm Res 1988; 5: 506508.

5. Olsson P, Aigbirhio F, Freemantle C, Pike V, Page P, Rhodes $\mathrm{C}$. The absorption and disposition of a non-chlorinated propellant. GR106642X (1,1,1,2-tetra-fluoroethane, HFA134a) as examined by 18 F-Iabelled and wholebody counting in healthy subjects. Am J Respir Crit Care Med 1994; 149: A220.

6. Kirby SM, Smith J, Ventresca GP. Salmeterol inhaler using a nonchlorinated propellant, HFA134a: systemic 
pharmacodynamic activity in healthy volunteers. Thorax 1995; 50: 679-681.

7. Denyer L, Kirby S, Olsson P, Ventresca G. HFA134a, a nonchlorinated propellant for use in metered-dose inhalers: Safety, tolerability and blood pharmacokinetics in healthy volunteers. Br J Clin Pharmacol 1994; 37: 509.

8. Maesen FP, Greefhorst LP, Smeets JJ, Wald FD, Cornelissen PJ. Therapeutic equivalence of a novel HFA134acontaining metered-dose inhaler and the conventional CFC inhaler (Berodual) for the delivery of a fixed combination of fenoterol/ipratropium bromide. A randomized double-blind placebo-controlled crossover study in patients with asthma. Respiration 1997; 64: 273-280.

9. Aubier M, Iacono P, Jirou-Najou J. Berodual HFA study group. Combination MDI containing fenoterol 50 microgram and ipratropium 20 microgram with HFA propellant is safe and effective during 3 months use in asthma and COPD. Eur Respir J 1997; 10: Suppl. 25, 240s.

10. Refabert L, Iacono P, Jirou-Najou JL, Scheinmann P, Berodual HFA study group. HFA formulation of fenoterol and ipratropium MDI is as safe and effective as the CFC formulation for long-term treatment of children with asthma. Eur Respir J 1997; 10: Suppl. 25, 221s.

11. Medicines Control Agency, Committee on Safety of Medicines, Royal College of General Practitioners, British Medical Association and Association of the British Pharmaceutical Industry. Guidelines for company-sponsored Safety Assessment of Marketed Medicines (SAMM) guidelines. Br J Clin Pharmacol 1994; 38: 95-97.

12. American Thoracic Society. Standards for the diagnosis and care of patients with chronic obstructive pulmonary disease (COPD) and asthma. Am Rev Respir Dis 1987; 136: 225-244.

13. Quanjer PH, Tammeling GJ, Cotes JE, Pedersen OF, Peslin R, Yernault JC. Lung volumes and forced ventilatory flows. Report Working Party Standardization of Lung Function Tests, European Community for Steel and Coal. Official Statement of the European Respiratory Society. Eur Respir J 1993; 16: 5-40.

14. American Thoracic Society. Standardization of Spirometry, 1994 Update. Am J Respir Crit Care Med 1995; 152: $1107-1136$. 